\title{
Novel Simulation Model of Non-Muscle Invasive Bladder Cancer: A Platform for a Virtual Randomized Trial of Conservative Therapy vs. Cystectomy in BCG Refractory Patients
}

\author{
Sanjay Patel ${ }^{\mathrm{a}, *}$, Tuan Dinh ${ }^{\mathrm{b}}$, Joyce Noah-Vanhoucke ${ }^{\mathrm{b}}$, Badri Rengarajan ${ }^{\mathrm{b}}$, Kevin Mayo ${ }^{\mathrm{c}}$, \\ Peter E. Clark ${ }^{\mathrm{d}}$, Ashish M. Kamat ${ }^{\mathrm{e}}$, Cheryl T. Lee ${ }^{\mathrm{f}}$, Wade J. Sexton ${ }^{\mathrm{g}}$ and Gary D. Steinberg ${ }^{\mathrm{a}}$ \\ ${ }^{a}$ University of Chicago, Chicago, IL, USA \\ $\mathrm{b}^{\mathrm{b}}$ Archimedes Inc., San Francisco, CA, USA \\ ${ }^{\mathrm{c}}$ Endo Pharmaceuticals, Newark, NJ, USA \\ ${ }^{\mathrm{d}}$ Vanderbilt University Medical Center, Nashville, TN, USA \\ ${ }^{\mathrm{e}}$ M.D. Anderson Cancer Center, Houston, TX, USA \\ ${ }^{\mathrm{f}}$ University of Michigan, Ann Arbor, MI, USA \\ ${ }^{\mathrm{g}}$ H. Lee Moffitt Cancer Center, Tampa Bay, FL, USA
}

\begin{abstract}
.
Introduction: There have been no randomized controlled trials (RCTs) evaluating the clinical or economic benefit of mitomycin $\mathrm{C}$ intravesical therapy vs. radical cystectomy in patients with high-risk non-muscle invasive bladder cancer (NMIBC). We used the Archimedes computational model to simulate RCT comparing radical cystectomy versus intravesical mitomycin C (MMC) therapy to evaluate the clinical and economic outcomes for BCG-refractory NMIBC as well demonstrate the utility of computer based models to simulate a clinical trial.

Methods: The Archimedes model was developed to generate a virtual population using the Surveillance Epidemiology and End Results database, other clinical trials, and expert opinions. Patients selected were diagnosed with NMIBC ( $<$ cT2 disease) who recurred or progressed despite BCG therapy and were randomized to 1) immediate radical cystectomy vs. 2) MMC induction intravesical therapy. Clinical (progression, overall survival, and disease specific survival) and economic outcomes were reported. Results: A total of 1300 virtual patients were evaluation. Progression to MIBC in the MMC treatment arm was $30 \%$ over the lifetime. Disease specific death at 5 years was $1.6 \%$ and $8.7 \%$ for the immediate cystectomy and MMC treatment arms respectively; while, overall death was $17.8 \%$ and $23.8 \%$ at 5 years. Over a 5 -year period the average cost of immediate cystectomy was $\$ 64,675$ vs $\$ 68,517$ in the MMC arm.
\end{abstract}

Keywords: Bladder Cancer, BCG failure, virtual clinical trial, mitomycin C

\footnotetext{
${ }^{*}$ Correspondence to: Sanjay Patel, University of Chicago Medical Center, Department of Surgery, Section of Urology, 5841 South Maryland Avenue - MC6038 Chicago, IL 60637, USA.
}

Tel.: +1 773702 3080; Fax: +1 615322 8990; E-mail: Sanjay.Patel@ uchospitals.edu. 
Conclusion: Immediate radical cystectomy after BCG failure for NMIBC has improved survival and is more cost-effective when compared to those undergoing MMC. Simulation of clinical trials using computational models similar to the Archimedes model can overcome shortcomings of real-world clinical trials and may prove useful in the face of current medical cost-conscious era.

\section{INTRODUCTION}

High risk (high grade, cTa, cT1, and cTis), nonmuscle invasive bladder cancer (NMIBC) is principally managed with transurethral resections (TUR) and adjuvant intravesical bacillus Calmette-Guerin (BCG) therapy. However, disease recurs or progresses in approximately $40 \%-50 \%$ of patients [1]. Available management options for patients with recurrent or progressive high grade NMIBC include radical cystectomy ( $\mathrm{RC}$ ), additional intravesical therapy typically with chemotherapy, and clinical trial entry [2]. Some advocate $\mathrm{RC}$ in lieu of additional intravesical therapy as BCG failure portends poor oncologic outcomes when treated with bladder preservation and longterm survival in patients undergoing cystectomy with cTa/T1/Tis disease ranges from $80 \%-88 \%$ [3, 4]. Furthermore, cystectomy may be more cost-effective than intravesical therapy when used as first line treatment in certain high risk settings of NMIBC [5].

Mitomycin C (MMC) is predominantly utilized as treatment for patients with intermediate and high risk NMIBC as well as for patients who recur or progress despite intravesical BCG therapy [6]. There have been no randomized controlled trials (RCTs) evaluating the clinical or economic benefit of mitomycin $\mathrm{C}$ intravesical therapy over radical cystectomy in high risk NMIBC populations largely due to the expected challenges in patient accrual, real ethical concerns, expected costs of such a trial, and the willingness of investigators to participate [7].

In response to the difficulty in conducting RCTs, virtual clinical trials, which utilize computer algorithms to simulate clinical outcomes, have been under development [8]. The Archimedes Model, a large-scale integrated simulation model of human physiology, diseases, interventions and healthcare systems, has the ability to incorporate co-morbid conditions as well as human behavior (i.e. compliance with treatments, maintaining physician appointments etc) into medical models [8]. The Archimedes model has previously been utilized to study cost effectiveness of breast cancer and colorectal cancer screening $[9,10]$. Using the Archimedes model we simulated an RCT comparing radical cystectomy versus intravesical MMC therapy to evaluate the clinical and economic outcomes for BCGrefractory patients with NMIBC and to demonstrate the utility of computer based models to simulate a clinical trial.

\section{METHODS}

We evaluated the cost-effectiveness of treatments for BCG-refractory bladder cancer patients using the Archimedes Model, a large-scale integrated simulation model of human physiology, diseases, interventions and healthcare systems [8]. The core of the Archimedes Model is a set of mathematical equations that represent physiological outcomes at the clinical level (i.e., at the level of detail of basic medical tests, clinical trials, and patient charts) and care utilization in the health care system. Currently, the Archimedes Model integrates several medical conditions including diabetes, congestive heart failure, coronary artery disease, stroke, hypertension, obesity, asthma, COPD and cancers of the breast, lung, colon and bladder. The Archimedes Model furthermore has the ability to factor in co-morbid conditions into the model as well as incorporating aspects of human behavior (i.e. compliance with treatments, maintaining physician appointments etc).

The NMIBC model was developed by at team at Archimedes with a panel of bladder cancer experts and Endo Pharmaceuticals Inc. The model integrates information from many data sources including: Surveillance Epidemiology and End Results (SEER) database, patient level data from the Urocidin ${ }^{\mathrm{TM}}$ clinical trial, meta-analyses from clinical trials, large retrospective datasets and expert opinions into an integrated framework Fig. 1. The supplementary appendix further details the components and assumptions made in the NMIBC model. The NMIBC model consists of the following components:

1) Patient generation: Virtual patient population, which is constructed from SEER case listings

2) Natural history: Natural history of NMIBC which captures recurrence, progression and survival. Figure 2 demonstrates pathways for tumor progression used in the model. 


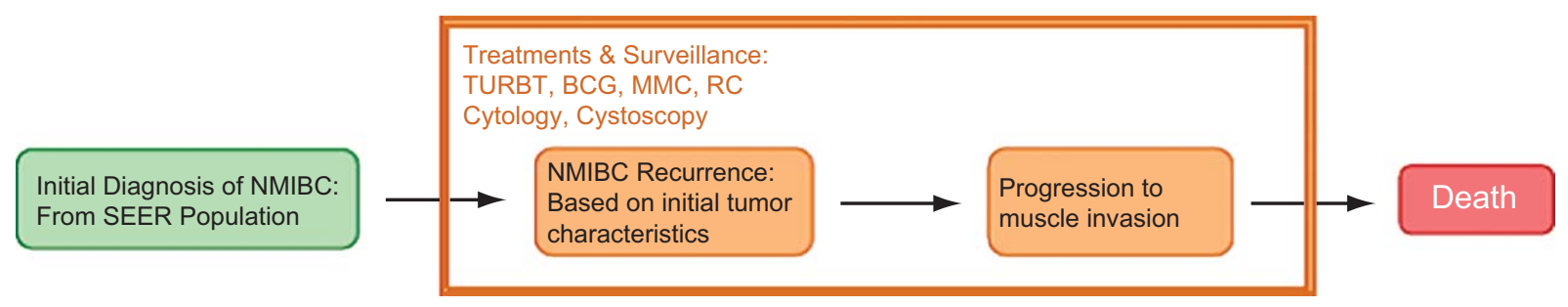

Fig. 1. Clinical Progression Framework.

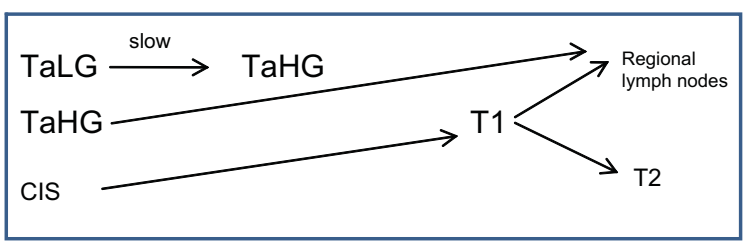

Fig. 2. Pathways for Tumor Progression in Non-muscle Invasive Bladder Cancer. LG - Low Grade, HG - High Grade, CIS - Carcinoma in Situ.

3) Intervention: Health care systems which captures interactions between patients and the health care system, clinical practices, and care interventions (drugs, diagnostic tests, etc)

4) Costs: tracks costs of procedures, tests, and treatments related to bladder cancer, thereby enabling economic analyses

\section{Virtual trial setup}

\section{Population generation}

Virtual NMIBC patients were constructed using SEER case listings. After initial diagnosis of NMIBC, patients were treated with standard of care as defined by the 2011 National Comprehensive Cancer Network (NCCN) guidelines and were given appropriate intravesical therapy based on NCCN guidelines. Patients selected for inclusion into the study were diagnosed with high risk NMIBC ( $<\mathrm{cT} 2$ disease) who recurred (at least one bladder tumor of the same or lower histological grade and T-stage as the primary (initial) tumor) or progressed (diagnosis of a new tumor within the bladder that is of higher histological grade or T-stage than the primary tumor) despite BCG therapy. BCG recurrence was defined as tumor recurrence of high-grade cTa, cT1, or Tis NMIBC within 1 year of BCG therapy. All patients were assumed to have received intravesical therapy in accordance with the recommendations of the 2011 NCCN guidelines.

\section{Trial arms}

Simulated patients who met inclusion criteria were randomized to radical cystectomy or MMC induction intravesical therapy. Patients who were randomized to the MMC arm underwent surveillance and follow-up as recommended by the NCCN guidelines and may have undergone cystectomy if they developed either a new tumor of any stage or muscle invasive bladder cancer. We assumed $100 \%$ compliance to the treatment arms defined and followed patients until death.

\section{Costs}

The cost of tests, procedures, and treatments were based on Medicare reimbursement rates. A payor perspective was used with costs discounted by $3 \%$. Results of the clinical trial simulation are imported into the Archimedes Outcomes Analyzer, a data-viewing tool that allows users to adjust certain parameters, such as costs and patient compliance. For the present study parameters included: population size, discount rate, and the cost of TURBT, cytology, cystoscopy, cystectomy, and intravesical therapies. The changes in health outcomes, cost and cost-effectiveness were performed. A separate analysis was performed that took into account the time value of money with a discount rate of $3 \%$.

\section{RESULTS}

A total of 1300 virtual patients were eligible for the trial with a mean age of 69 and $73 \%$ of patients being male. Table 1 displays a summary of demographic and tumor characteristics. At initial diagnosis $51 \%$ of patients had cT1 disease, $46 \%$ of patients had more than one tumor, and $19 \%$ had concomitant CIS. Prior to the start of the virtual trial, $58 \%$ of patient had cT1 as 
Table 1

Patient characteristics

\begin{tabular}{|c|c|c|c|}
\hline Risk factors & & & Virtual trial \\
\hline \multirow[t]{3}{*}{ Demographics } & Population size & & 1300 \\
\hline & $\begin{array}{l}\text { Average age at } \\
\text { primary diagnosis } \\
\text { of NMIBC (years) }\end{array}$ & & 69 \\
\hline & Male fraction & & $73 \%$ \\
\hline \multirow{5}{*}{$\begin{array}{l}\text { Tumor characteristics } \\
\text { at initial } \\
\text { diagnosis of NMIBC }\end{array}$} & Distribution of & $\mathrm{Ta}$ & $39 \%$ \\
\hline & T-stage at & Tis & $10 \%$ \\
\hline & primary diagnosis & $\mathrm{T} 1$ & $51 \%$ \\
\hline & $\begin{array}{l}\text { Fraction of patients } \\
\text { with concomitant CIS } \\
\text { at primary diagnosis }\end{array}$ & & $19 \%$ \\
\hline & $\begin{array}{l}\text { Fraction of } \\
\text { patients with } 2 \\
\text { or more tumors }\end{array}$ & & $46 \%$ \\
\hline \multirow[t]{5}{*}{ Disease history } & Worst T-stage prior to trial & TaHG & $22 \%$ \\
\hline & start (including tumors found & Tis & $20 \%$ \\
\hline & at the last TURBT) & $\mathrm{T} 1$ & $58 \%$ \\
\hline & $\begin{array}{l}\text { Average number of } \\
\text { tumor recurrences } \\
\text { prior trial start }\end{array}$ & & 1.8 \\
\hline & $\begin{array}{l}\text { Average time form } \\
\text { previous tumor recurrence } \\
\text { to trial start (years) }\end{array}$ & & 0.4 \\
\hline \multirow{5}{*}{$\begin{array}{l}\text { Characteristics of tumors } \\
\text { found by the last TURBT } \\
\text { prior to trial start }\end{array}$} & T-stage of tumors & TaHG & $49 \%$ \\
\hline & & CIS & $37 \%$ \\
\hline & & $\mathrm{T} 1$ & $14 \%$ \\
\hline & 2 or more tumors & & $14 \%$ \\
\hline & Tumor size & $>3 \mathrm{~cm}$ & $46 \%$ \\
\hline
\end{tabular}

Table 2

Clinical outcomes for cystectomy and conservative treatment arms

\begin{tabular}{llcc}
\hline Outcomes (per persons) & Immediate & $\begin{array}{c}\text { Conservative } \\
\text { cystectomy arm }\end{array}$ & $\begin{array}{c}\text { arm } \\
\text { treatment }\end{array}$ \\
\hline Cumulative incidence of progression to MIBC & Year 1 & $0 \%$ & $22 \%$ \\
& Year 5 & $0 \%$ & $27 \%$ \\
Cumulative incidence of bladder cancer death & Life time & $0 \%$ & $30 \%$ \\
& Year 1 & $0.8 \%$ & $3.6 \%$ \\
Cumulative incidence of death & Year 5 & $1.6 \%$ & $8.7 \%$ \\
& Life time & $1.9 \%$ & $14.2 \%$ \\
& Year 1 & $4.5 \%$ & $7.26 \%$ \\
Cumulative incidence of cystctomy & Year 5 & $17.8 \%$ & $23.8 \%$ \\
& Life time & $100 \%$ & $100 \%$ \\
Cumulative bladder cancer costs (not discounted) & Year 1 & $100 \%$ & $52.7 \%$ \\
& Year 5 & $100 \%$ & $62.8 \%$ \\
Total costs of surveillance of NMIBC & Year 30 & $100 \%$ & $68.8 \%$ \\
(TURBT, cytology and cystoscopy) & Year 1 & $\$ 64,083$ & $\$ 55,265$ \\
& Year 5 & $\$ 64,675$ & $\$ 68,517$ \\
Total costs of intravesical therapies (MMC and BCG) & Life time & $\$ 64,798$ & $\$ 77,174$ \\
& Year 1 & $\$ 3,086$ & $\$ 5,886$ \\
Treatment and bladder cancer death & Year 5 & $\$ 3,086$ & $\$ 7,590$ \\
& Life time & $\$ 3,086$ & $\$ 9,407$ \\
\hline & Year 1 & $\$ 0$ & $\$ 1,791$
\end{tabular}




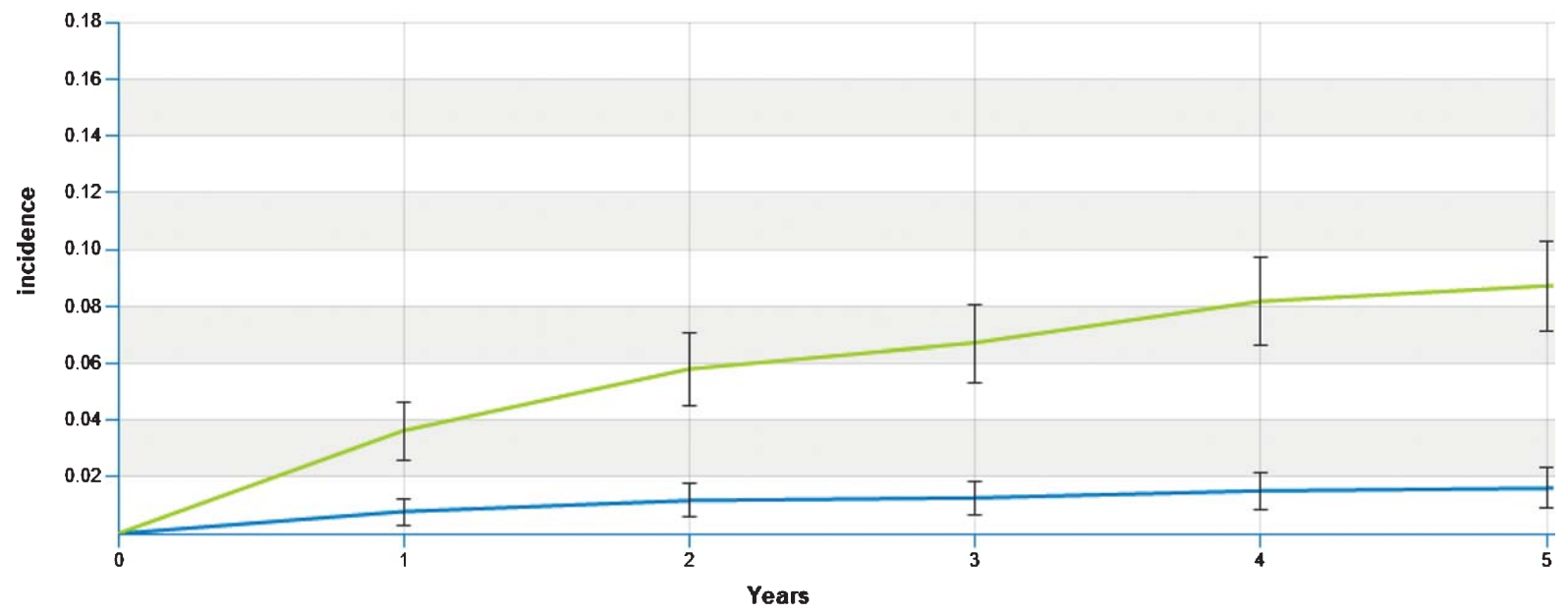

All:Everyone | Cystectomy | BladderCancerDeath (Kaplan-Meier)

All:Everyone | MMC | BladderCancerDeath (Kaplan-Meier)

Fig. 3. Cumulative risk of bladder cancer death.

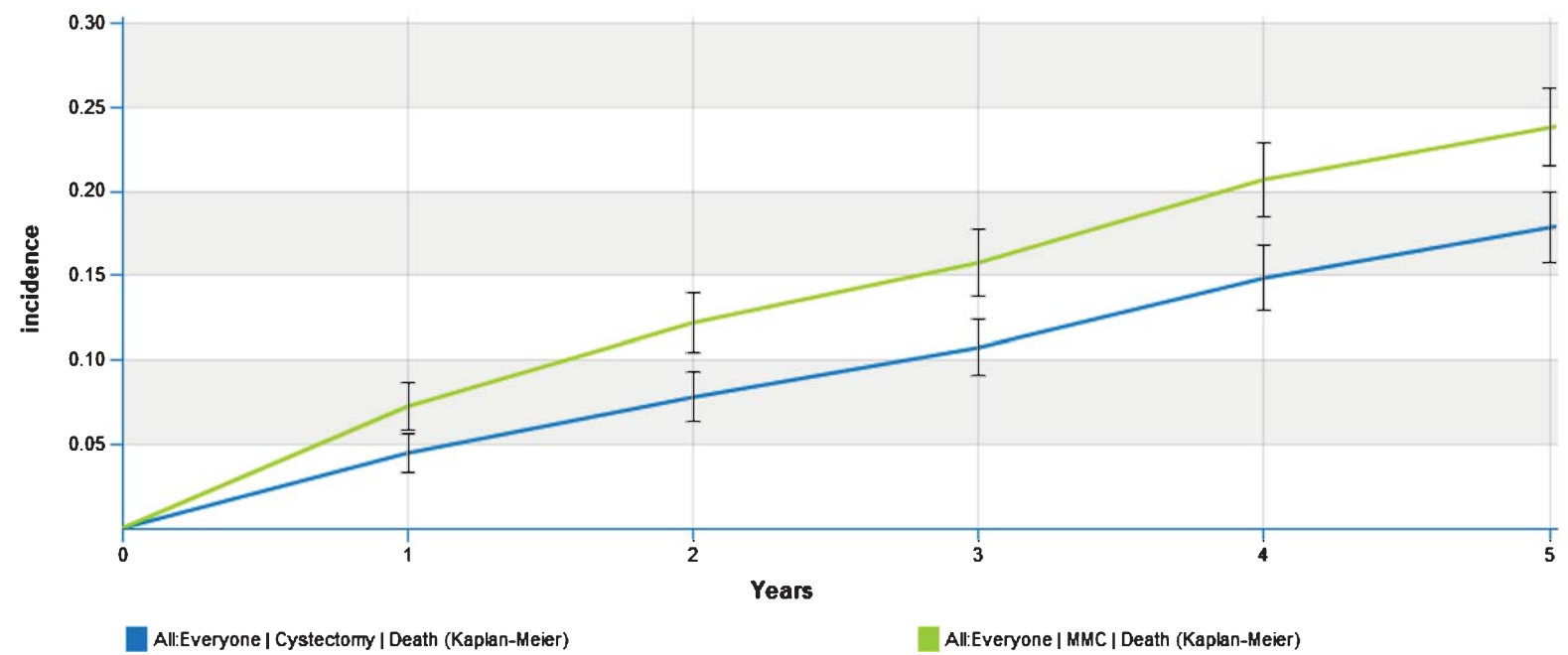

Fig. 4. Cumulative risk of overall death.

their worse cTstage on TURBT with an average of 1.8 tumor recurrences, and an average tumor recurrence time of 0.4 years.

\section{Clinical outcomes}

Clinical outcomes for the immediate cystectomy and the conservative MMC treatment arms are summarized in Table 2. The progression to MIBC in the conservative treatment arm was $22 \%$ at year $1,24 \%$ at year 3 and $27 \%$ at year 5, with an overall rate of $30 \%$ over the lifetime. The cumulative incidence of cystectomy in the conservative arm was $52.7 \%$ in year 1 and $62.8 \%$ at year 5. Disease specific death at 5 years was $1.6 \%$ and $8.7 \%$ for the immediate cystectomy and conservative treatment arms respectively; while, overall death was $17.8 \%$ and $23.8 \%$ at 5 years (Figs. 3 and 4 ). The lifetime risk of bladder cancer death of $14.2 \%$ vs. $1.9 \%$ in the MMC and $\mathrm{RC}$ arms respectively.

\section{Economic outcomes}

Over a 5-year period the average cost of cystectomy was $\$ 64,675$, dominated by the cost of initial cystectomy; while, patients in the conservative arm incurred average total costs of $\$ 68,517$ at 5 years. The cost of intravesical therapy at 5 years in the conservative group was $\$ 2365$ at 5 years. Surveillance of NMIBC, which 
Table 3

Clinical and economic outcomes associated with bladder cancer between immediate cystectomy and conservative treatment

\begin{tabular}{ll}
\hline Difference in economic outcomes between immediate cystectomy arm and conservative arm and conservative treatment arm \\
\hline $\begin{array}{l}\text { Delay (in years) of cystectomy for patients undergoing cystectomy in the } \\
\text { conservative treatment arm }\end{array}$ & Mean: 1.1 \\
& Median: 0.3 \\
Average life years gained per person & 5 Year time horizon: 0.22 LY \\
& Lifetime: $1.4 \mathrm{LY}$ \\
Average discounted life years gained per person (3\% annually) & 5 year time horizon: 0.19 LY \\
& Lifetime: 0.94 LY \\
Average bladder cancer cost saved per person & 5 year time horizon: $\$ 3,842$ \\
& Lifetime: $\$ 12,375$ \\
Average discounted bladder cancer cost saved per person (3\% annually) & 5 year time horizon: $\$ 3,585$ \\
& Lifetime: $\$ 9,937$ \\
\hline
\end{tabular}

included TURBT at time of randomization, was $\$ 3086$ vs. $\$ 7590$ at 5 years for immediate cystectomy and conservative treatment arms respectively.

\section{Clinical-economic outcomes}

Table 3 summarizes the differences in clinicaleconomic outcomes. Over a 5-year time period immediate cystectomy saves 0.19 life years and $\$ 3585$ in total bladder cancer cost compared to the conservative arm (assuming a 3\% discount rate). Over a lifetime cystectomy saves 0.94 life years and $\$ 9937$.

\section{DISCUSSION}

We report on the first virtual clinical trial in the bladder cancer literature to date. Using the Archimedes model, 1300 BCG refractory patients were randomized to $\mathrm{RC}$ or MMC while factoring in co-morbid conditions, natural history of disease, treatment characteristics, and aspects of human behavior. We found that 1 in 3 patients progressed to MIBC in the MMC arm with a lifetime risk of bladder cancer death of $14.2 \%$ vs. $1.9 \%$ in the RC arm. Overall economic outcomes were more favorable in the $\mathrm{RC}$ group with lifetime saving of 0.94 life years and \$9937.

Due to the heterogeneity of and low number of clinical trials in BCG refractory patients, comparison of our study population with other series is difficult; however, the simulated study population has similar clinical characteristics to prior studies. The study population of 1300 virtual patients is significantly higher than most published series on BCG refractory patients which range from $20-100$ patients [11-15]. Our virtual series contained $58 \%$ of patients with clinical stage $\mathrm{T} 1$ urothelial carcinoma prior to randomization which falls within the range of previous BCG failure trials where $47 \%-78 \%$ had cT1 tumors $[11,15]$.
Thirty-seven percent of patients had cTis prior to the start of the virtual trial which falls within the range of $31 \%-77 \%$ from prior studies $[11,15]$.

There was no progression to muscle invasion in the immediate cystectomy group as it was assumed that there was no upstaging to muscle invasion; while, there was a $27 \%$ and $30 \%$ progression to muscle invasion at 5 years and over lifetime, respectively, for patients in the MMC group. This is lower than in the recent Phase II clinical trial comparing salvage intravesical gemcitabine (33\% progression) vs. additional induction BCG (37.5\% progression) in patients with recurrent NMIBC despite BCG at 2 years follow-up [11]. The rate of cystectomy was $62 \%$ at 5 years which is higher than previously published clinical trials which range from $33 \%$ to $56 \%$ at a follow-up of 1.5 to 3 years [11-15]. The differences are largely attributable to the fact that in our series we assumed perfect compliance and follow-up as well as strict adherence to NCCN guidelines, which are more aggressive than real-world settings.

The cumulative incidence of death for the RC group was $17.8 \%$ (82\% survival) at 5 years which is similar to previously reported studies which report 5 year survival of $80 \%-90 \%$ for patients who undergo RC with non-muscle invasive disease [4]. The cumulative incidence of death was higher $(24 \%)$ in the MMC group at 5 years and is certainly attributed to the $27 \%$ of patients who progress to MIBC. Overall, virtual patients in the present study have a more favorable survival compared with patients from other series. Lerner et al. performed a subgroup analysis of the SWOG 8507 trial for patients who did not completely respond to BCG therapy reporting 5 year overall survival rates of $33 \%$ and $50 \%$ for patients who were $>61$ years of age with prior intravesical chemotherapy vs. $>61$ years of age and no prior intravesical chemotherapy respectively [3]. 
Bladder cancer has been shown to be the most expensive cancer to treat from diagnosis to death because of the requirement of lifelong surveillance and treatment [16]. Kulkarni et al. conducted a cost-analysis of immediate RC vs. intravesical BCG therapy and demonstrated more favorable clinical outcomes and lower costs with immediate $\mathrm{RC}$ in healthy patients age of $<60$ years; while, the most cost effective therapy in patients aged $>70$ was initial intravesical therapy. The present series revealed a more favorable cost for immediate RC over MMC with $\$ 9937$ savings over a lifetime. Most of the costs associated with immediate $\mathrm{RC}$ were realized upfront at the time of cystectomy with an average cost of $\$ 61,997$ at year one. While the average age of the population was 70 years old, there was still a cost benefit with more aggressive therapy with RC.

While the Archmides model incorporated data from several established sources (SEER, Urocidin trial, BCG failure clinical trials), the results rely on computer algorithms, which are dependent on the manner in which the mathematical models were programmed and on various clinical assumptions made (i.e. performance of TURBT to remove all disease, efficacy of MMC etc.). The reproducibility of the results generated from such a computer-based model created by a different company/programming group would be difficult. The present study assumed perfect compliance and followup per NCCN guidelines, which are more aggressive than real-world settings and likely resulted in more favorable simulated clinical outcomes. In the immediate cystectomy group there it was assumed that there was no upstaging to muscle invasion, which may favorably bias the survival in this arm. Although cystectomy appears favored based on the clinical/economic data, we did not study impacts on QOL based health related outcomes (largely related to sexual dysfunction and urinary diversions).

With improved computing ability and processing technology, virtual trials such as the current study may be a supplement and possibly an alternative to performing traditional clinical trials, which can be cost-prohibitive, have poor accrual, and require a long duration for results. Perhaps virtual clinical trials could be performed as an initial feasibility assessment prior to dedicating time, effort, and financial resources for a dedicated RCT. This simulation lends support to utilizing the Archimedes model prior to undertaking any clinical treatment trial to assess the feasibility and likelihood of a clinically and statistically meaningful result. While results of this virtual trial seem promising, they would still need to be confirmed in a real world setting. Nonetheless, the simulation environment can overcome some of the shortcomings of real-world clinical trials such as the inability to have a placebo arm, incorrect enrollment of patients (e.g., due to sites not properly following inclusion/exclusion criteria), missing data, protocol deviations, failure to achieve expected accrual. Virtual trials can also help to fill in the "gaps" in the clinical trials landscape specifically for certain therapies not tested in the literature, elderly population or other populations not previously investigated, certain combinations of therapies not previously tested, and trials precluded due to ethical restrictions, different protocols/care guidelines, and cost.

\section{SUPPLEMENTARY MATERIAL}

The supplementary Appendix is available in the electronic version of this article: http://dx.doi.org/ 10.3233/BLC-150020.

\section{FUNDING}

None.

\section{REFERENCES}

[1] Babjuk M, Oosterlinck W, Sylvester R, et al. EAU guidelines on non-muscle-invasive urothelial carcinoma of the bladder, the 2011 update. European Urology 2011; 59:997-1008.

[2] Yates DR, Brausi MA, Catto JWF, et al. Treatment options available for bacillus Calmette-Guérin failure in nonmuscle-invasive bladder cancer. European Urology 2012;62: 1088-96.

[3] Lerner SP, Tangen CM, Sucharew H, et al. Failure to achieve a complete response to induction BCG therapy is associated with increased risk of disease worsening and death in patients with high risk non-muscle invasive bladder cancer. URO 2009;27:155-9.

[4] Stein JP, Lieskovsky G, Cote R, et al. Radical cystectomy in the treatment of invasive bladder cancer: Long-term results in 1,054 patients. Journal of Clinical Oncology 2001;19: 666-75.

[5] Kulkarni GS, Alibhai SMH, Finelli A, et al. Cost-effectiveness analysis of immediate radical cystectomy versus intravesical Bacillus Calmette-Guerin therapy for high-risk, high-grade (T1G3) bladder cancer. Cancer 2009;115:5450-9.

[6] Sylvester RJ, Oosterlinck W, van der Meijden APM. A single immediate postoperative instillation of chemotherapy decreases the risk of recurrence in patients with stage Ta T1 bladder cancer: A meta-analysis of published results of randomized clinical trials. The Journal of Urology 2004;171:2186-90.

[7] Malmström PU, Wijkström H, Lundholm C, et al. 5-year followup of a randomized prospective study comparing mitomycin $\mathrm{C}$ and bacillus Calmette-Guerin in patients with superficial bladder carcinoma. Swedish-Norwegian Bladder Cancer Study Group. JURO 1999;161:1124-7. 
[8] Schlessinger L, Eddy DM. Archimedes: A new model for simulating health care systems - the mathematical formulation. Journal of Biomedical Informatics 2002;35(1):37-50.

[9] Dinh TA, Rosner BI, Atwood JC, et al. Health benefits and cost-effectiveness of primary genetic screening for Lynch syndrome in the general population. Cancer Prevention 2011;4(1):9-22.

[10] Noah-Vanhoucke J, Green LE, Dinh TA, et al. Costeffectiveness of chemoprevention of breast cancer using tamoxifen in a postmenopausal US population. Cancer 2011;117:3322-31.

[11] Di Lorenzo G, Perdonà S, Damiano R, et al. Gemcitabine versus bacille Calmette-Guérin after initial bacille CalmetteGuérin failure in non-muscle-invasive bladder cancer. Cancer 2010;116:1893-900.

[12] Gunelli R, Bercovich E, Nanni O, et al. Activity of endovesical gemcitabine in BCG-refractory bladder cancer patients: A translational study. Br J Cancer 2007;97:1499-504
[13] Steinberg G, Bahnson R, Brosman S, et al. Efficacy and safety of valrubicin for the treatment of Bacillus Calmette-Guerin refractory carcinoma in situ of the bladder. The Valrubicin Study Group. JURO 2000;163:761-7.

[14] Gacci M, Bartoletti R, Cai T, et al. Intravesical Gemcitabine in BCG-Refractory T1G3 Transitional Cell Carcinoma of the Bladder: A Pilot Study. Urologia Internationalis 2006;76: 106-11.

[15] Dalbagni G, Russo P, Bochner B, et al. Phase II trial of intravesical gemcitabine in bacille Calmette-Guérin-refractory transitional cell carcinoma of the bladder. J Clin Oncol 2006; 24(18):2729-34

[16] Botteman MF, Pashos CL, Redaelli A, et al. The health economics of bladder cancer: A comprehensive review of the published literature. Pharmacoeconomics 2003;21:1315-30. 